\title{
Existence Results for Nonlinear Multiorder Fractional Differential Equations with Integral and Antiperiodic Boundary Conditions
}

\author{
HuiChol Choi, YongSim Sin, and KumSong Jong $(\mathbb{D}$ \\ Faculty of Mathematics, Kim Il Sung University, Pyongyang, Democratic People's Republic of Korea
}

Correspondence should be addressed to KumSong Jong; ksjong1016@163.com

Received 3 February 2020; Accepted 21 March 2020; Published 16 April 2020

Academic Editor: Yun-Bo Zhao

Copyright ( 2020 HuiChol Choi et al. This is an open access article distributed under the Creative Commons Attribution License, which permits unrestricted use, distribution, and reproduction in any medium, provided the original work is properly cited.

\begin{abstract}
In this paper, we study the solvability of a class of nonlinear multiorder Caputo fractional differential equations with integral and antiperiodic boundary conditions. By using some fixed point theorems including the Banach contraction mapping principle and Schaefer's fixed point theorem, we obtain new existence and uniqueness results for our given problem. Also, we give some examples to illustrate our main results.
\end{abstract}

\section{Introduction}

Fractional calculus has a history of several hundred years, and many valuable results that have contributed to the development of mathematical theories and their application to practice have been created during its historical process (see [1]). Also, fractional differential equations are one of the powerful means to model and solve scientific and technological problems that have been arisen in physics, chemistry, biology, mechanics, and many other fields, and it has developed more and more in-depth (see [2]). In particular, boundary value problems of fractional differential equations are often used as mathematical models for many phenomena in a variety of physical, biological, mechanical, and chemical studies such as analysis of turbulent flow, simulation of chemical reaction, and image processing technique (see [3-6]).

In recent years, antiperiodic boundary value problems have been put forward in various practical phenomena and have attracted the attention of a large number of researchers because of the specific properties of their solutions (see [7]). Based on many works about the solvability of antiperiodic boundary value problems for integer-order differential equations (see [8-10]), a lot of attempts have been made to extend the existence results for them to the case of fractional differential equations (see [11-20]). For instance, Agarwal and
Ahmad [11] established the existence of solutions of the following single-term Caputo fractional differential equations with antiperiodic boundary conditions by using the nonlinear alternative of Leray-Schauder type and Leray-Schauder degree theory:

$$
\left\{\begin{array}{l}
{ }^{c} D^{q} x(t)=f(t, x(t)), \quad t \in[0, T], T>0,3<q \leq 4, \\
x(0)=-x(T), x^{\prime}(0)=-x^{\prime}(T), x^{\prime \prime}(0)=-x^{\prime \prime}(T), x^{\prime \prime \prime}(0)=-x^{\prime \prime \prime}(T) .
\end{array}\right.
$$

In [17], Choudhary and Daftardar-Gejji considered the antiperiodic boundary value problem of the nonlinear multiorder Caputo fractional differential equation

$$
\left\{\begin{array}{l}
\sum_{i=0}^{n} \lambda_{i}^{c} D^{\alpha_{i}} u(t)=f(t, u(t)), \quad t \in[0, T] \\
u(0)=-u(T)
\end{array}\right.
$$

where $\quad \lambda_{i} \in \mathbf{R}, i=0,1, \cdots, n, \lambda_{n} \neq 0,0 \leq \alpha_{0}<\alpha_{1}<\cdots<\alpha_{n}<1$. They proved the existence and uniqueness of solutions to their problem in terms of the two-parametric functions of Mittag-Leffler type. Their equation in problem (2) is a 
generalization of the classical relaxation equation and governs some fractional relaxation processes.

Analyzing the higher-order fractional differential equations like that in problem (1), some new research papers considered not only antiperiodic boundary conditions but also mixed-type boundary conditions which are composed of both integral and antiperiodic boundary conditions (see [21-25]). Xu [24] obtained new existence and uniqueness results for the following single-term fractional differential equations with integral and antiperiodic boundary conditions by means of the Krasnosel'skii fixed point theorem, contraction mapping principle, and Leray-Schauder degree theory:

$$
\left\{\begin{array}{l}
{ }^{c} D^{q} x(t)=f(t, x(t)), \quad t \in[0,1], 1<q<2, \\
x(1)=\mu \int_{0}^{1} x(s) d s, x^{\prime}(0)+x^{\prime}(1)=0
\end{array}\right.
$$

Taking the previous results together, we can know that very little has been done on the multiterm fractional differential equations with integral and antiperiodic boundary conditions. In particular, as far as we know, the research on the mixed-type boundary value problems of nonlinear multiorder fractional differential equations like that in problem (2) which is of great significance in practice has not been carried out at all.

Motivated by above analysis, in this paper, we investigate the existence and uniqueness of solutions for the following mixed-type fractional boundary value problems of combining the nonlinear multiorder Caputo fractional differential equations, which are similar to the equation in problem (2) and have higher fractional orders, with the integral and antiperiodic boundary conditions in problem (3):

$$
\begin{gathered}
{ }^{c} D_{0+}^{\alpha} u(t)+\sum_{i=1}^{n} \lambda_{i}(t)^{c} D_{0+}^{\alpha_{i}} u(t)+\sum_{i=1}^{m} \mu_{i}(t)^{c} D_{0+}^{\beta_{i}} u(t) \\
+\sigma(t) u(t)=f(t, u(t)), \quad t \in[0,1], \\
u(1)=\mu \int_{0}^{1} u(s) d s, \\
u^{\prime}(0)+u^{\prime}(1)=0,
\end{gathered}
$$

$$
G(t, s)= \begin{cases}\frac{(t-s)^{\alpha-1}}{\Gamma(\alpha)}+\frac{\mu}{4(1-\mu) \Gamma(\alpha+1)}\left[4(1-s)^{\alpha}-4 \alpha(1-s)^{\alpha-1}+\alpha(\alpha-1)(1-s)^{\alpha-2}\right]+\frac{1}{2 \Gamma(\alpha)}\left[(1-t)(\alpha-1)(1-s)^{\alpha-2}-2(1-s)^{\alpha-1}\right], & 0 \leq s \leq t \leq 1 \\ \frac{\mu}{4(1-\mu) \Gamma(\alpha+1)}\left[4(1-s)^{\alpha}-4 \alpha(1-s)^{\alpha-1}+\alpha(\alpha-1)(1-s)^{\alpha-2}\right]+\frac{1}{2 \Gamma(\alpha)}\left[(1-t)(\alpha-1)(1-s)^{\alpha-2}-2(1-s)^{\alpha-1}\right], & 0 \leq t \leq s \leq 1 .\end{cases}
$$

where $1<\alpha_{1}<\cdots<\alpha_{n}<\alpha \leq 2,0<\beta_{1}<\cdots<\beta_{m}<1,0<\mu<1$, $\lambda_{i} \in C[0,1](i=1,2, \cdots, n), \mu_{i} \in C[0,1](i=1,2, \cdots, m), \sigma \in C[0$ $, 1], f \in C([0,1] \times \mathbf{R}, \mathbf{R})$.

\section{Derivation of the Integral Equation}

The Riemann-Liouville fractional integral and the Caputo fractional derivative of order $\alpha>0$ of a function $f:(0, \infty) \longrightarrow \mathbf{R}$ are given by

$$
\begin{aligned}
\left(I_{0+}^{\alpha} f\right)(t) & :=\frac{1}{\Gamma(\alpha)} \int_{0}^{t}(t-s)^{\alpha-1} f(s) d s \\
\left({ }^{c} D_{0+}^{\alpha} f\right)(t) & :=\frac{1}{\Gamma(n-\alpha)} \int_{0}^{t}(t-s)^{n-\alpha-1} f^{(n)}(s) d s,
\end{aligned}
$$

where $n=[\alpha]+1$, provided that the right-hand sides are pointwise defined on $(0, \infty)$ (see [1]).

Definition 1. A function $u \in C[0,1]$ with a Caputo fractional derivative of order $\alpha$ that belongs to $C[0,1]$ (i.e., ${ }^{c} D_{0+}^{\alpha} u \in C[$ $0,1])$ is said to be a solution of problem (4)-(5) if it satisfies equation (4) and the boundary conditions (5).

Lemma 2. If a function $u$ is a solution of problem (4)-(5), then $y(t):={ }^{c} D_{0+}^{\alpha} u(t)$ is a solution of the integral equation

$$
\begin{aligned}
y(t)=f( & \left.t, \int_{0}^{1} G(t, s) y(s) d s\right)-\sum_{i=1}^{n} \lambda_{i}(t) I_{0+}^{\alpha-\alpha_{i}} y(t)-\sum_{i=1}^{m} \mu_{i}(t) I_{0+}^{\alpha-\beta_{i}} y(t) \\
& +\left.\frac{1}{2} \sum_{i=1}^{m} \frac{\mu_{i}(t) \cdot t^{1-\beta_{i}}}{\Gamma\left(2-\beta_{i}\right)} I_{0+}^{\alpha-1} y(t)\right|_{t=1}-\sigma(t) \int_{0}^{1} G(t, s) y(s) d s,
\end{aligned}
$$

in $C[0,1]$, and conversely, if $y \in C[0,1]$ is a solution of the integral equation (7), then a function $u$ which is given by

$$
u(t)=\int_{0}^{1} G(t, s) y(s) d s
$$

is a solution of problem (4)-(5), where 
Proof. Let a function $u$ be a solution of problem (4)-(5). Applying $I_{0+}^{\alpha}$ on both sides of the expression $y(t)={ }^{c} D_{0+}^{\alpha} u(t)$, it is obvious that

$$
u(t)=\int_{0}^{1} G(t, s) y(s) d s
$$

(see [24]).

We can rewrite (10) as

$$
\begin{aligned}
u(t)= & \left.\frac{\mu}{1-\mu} I_{0+}^{\alpha+1} y(t)\right|_{t=1}-\left.\frac{\mu}{1-\mu} I_{0+}^{\alpha} y(t)\right|_{t=1}+\left.\frac{\mu}{4(1-\mu)} I_{0+}^{\alpha-1} y(t)\right|_{t=1} \\
& +I_{0+}^{\alpha} y(t)-\left.I_{0+}^{\alpha} y(t)\right|_{t=1}+\left.\frac{1}{2} I_{0+}^{\alpha-1} y(t)\right|_{t=1} \\
& -\left.\frac{t}{2} I_{0+}^{\alpha-1} y(t)\right|_{t=1}=I_{0+}^{\alpha} y(t)+\left.\frac{\mu}{1-\mu} I_{0+}^{\alpha+1} y(t)\right|_{t=1} \\
& -\left.\frac{1}{1-\mu} I_{0+}^{\alpha} y(t)\right|_{t=1}+\left.\left(\frac{\mu}{4(1-\mu)}+\frac{1-t}{2}\right) I_{0+}^{\alpha-1} y(t)\right|_{t=1}
\end{aligned}
$$

From (11), we have some equalities as follows:

$$
\begin{aligned}
& { }^{c} D_{0+}^{\alpha_{i}} u(t)=I_{0+}^{\alpha-\alpha_{i}} y(t), \quad i=1, \cdots, n, \\
& { }^{c} D_{0+}^{\beta_{i}} u(t)=I_{0+}^{\alpha-\beta_{i}} y(t)-\left.\frac{1}{2 \Gamma\left(2-\beta_{i}\right)} \cdot t^{1-\beta_{i}} I_{0+}^{\alpha-1} y(t)\right|_{t=1}, \quad i=1, \cdots, m .
\end{aligned}
$$

Substituting the above ${ }^{c} D_{0+}^{\alpha_{i}} u(t)$ and ${ }^{c} D_{0+}^{\beta_{i}} u(t)$ into (4), it can be easily seen that

$$
\begin{aligned}
y(t) & +\sum_{i=1}^{n} \lambda_{i}(t) I_{0+}^{\alpha-\alpha_{i}} y(t) \\
& +\sum_{i=1}^{m} \mu_{i}(t)\left(I_{0+}^{\alpha-\beta_{i}} y(t)-\left.\frac{1}{2 \Gamma\left(2-\beta_{i}\right)} \cdot t^{1-\beta_{i}} I_{0+}^{\alpha-1} y(t)\right|_{t=1}\right) \\
& +\sigma(t) \int_{0}^{1} G(t, s) y(s) d s=f\left(t, \int_{0}^{1} G(t, s) y(s) d s\right)
\end{aligned}
$$

This yields the integral equation (7).

Conversely, let a function $y \in C[0,1]$ be a solution of the integral equation (7). Substituting the expression

$$
u(t)=\int_{0}^{1} G(t, s) y(s) d s
$$

into (7), we can get that

$$
\begin{aligned}
y(t) & +\sum_{i=1}^{n} \lambda_{i}(t) I_{0+}^{\alpha-\alpha_{i}} y(t) \\
& +\sum_{i=1}^{m} \mu_{i}(t) I_{0+}^{\alpha-\beta_{i}} y(t)-\left.\frac{1}{2} \sum_{i=1}^{m} \frac{\mu_{i}(t) \cdot t^{1-\beta_{i}}}{\Gamma\left(2-\beta_{i}\right)} I_{0+}^{\alpha-1} y(t)\right|_{t=1} \\
& +\sigma(t) u(t)=f(t, u(t)) .
\end{aligned}
$$

On the other hand, using the expression of Green's function $G(t, s)$, we can see that

$$
\begin{aligned}
u(t)= & I_{0+}^{\alpha} y(t)+\left.\frac{\mu}{1-\mu} I_{0+}^{\alpha+1} y(t)\right|_{t=1} \\
& -\left.\frac{1}{1-\mu} I_{0+}^{\alpha} y(t)\right|_{t=1}+\left.\left(\frac{\mu}{4(1-\mu)}+\frac{1-t}{2}\right) I_{0+}^{\alpha-1} y(t)\right|_{t=1} .
\end{aligned}
$$

So, we know that ${ }^{c} D_{0+}^{\alpha} u(t),{ }^{c} D_{0+}^{\alpha_{i}} u(t)$, and ${ }^{c} D_{0+}^{\beta_{i}} u(t)$ exist for any $t \in[0,1]$ and ${ }^{c} D_{0+}^{\alpha} u \in C[0,1]$.

Considering the relations

$$
\begin{aligned}
& { }^{c} D_{0+}^{\alpha_{i}} u(t)=I_{0+}^{\alpha-\alpha_{i}} y(t), \quad i=1, \cdots, n, \\
& { }^{c} D_{0+}^{\beta_{i}} u(t)=I_{0+}^{\alpha-\beta_{i}} y(t)-\left.\frac{1}{2 \Gamma\left(2-\beta_{i}\right)} \cdot t^{1-\beta_{i}} I_{0+}^{\alpha-1} y(t)\right|_{t=1}, \quad i=1, \cdots, m,
\end{aligned}
$$

we can rewrite (15) as

$$
\begin{aligned}
{ }^{c} D_{0+}^{\alpha} u(t) & +\sum_{i=1}^{n} \lambda_{i}(t)^{c} D_{0+}^{\alpha_{i}} u(t)+\sum_{i=1}^{m} \mu_{i}(t)^{c} D_{0+}^{\beta_{i}} u(t) \\
& +\sigma(t) u(t)=f(t, u(t)) .
\end{aligned}
$$

That is, $u$ satisfies equation (4).

Now, we prove that $u$ satisfies the boundary conditions (5). By simple calculation, we have

$$
\begin{aligned}
u(1)= & \left.\frac{\mu}{1-\mu} I_{0+}^{\alpha+1} y(t)\right|_{t=1}-\left.\frac{\mu}{1-\mu} I_{0+}^{\alpha} y(t)\right|_{t=1} \\
& +\left.\frac{\mu}{4(1-\mu)} I_{0+}^{\alpha-1} y(t)\right|_{t=1}+\left.I_{0+}^{\alpha} y(t)\right|_{t=1} \\
& -\left.I_{0+}^{\alpha} y(t)\right|_{t=1}+\left.\frac{1}{2} I_{0+}^{\alpha-1} y(t)\right|_{t=1} \\
& -\left.\frac{1}{2} I_{0+}^{\alpha-1} y(t)\right|_{t=1}=\left.\frac{\mu}{1-\mu} I_{0+}^{\alpha+1} y(t)\right|_{t=1} \\
& -\left.\frac{\mu}{1-\mu} I_{0+}^{\alpha} y(t)\right|_{t=1}+\left.\frac{\mu}{4(1-\mu)} I_{0+}^{\alpha-1} y(t)\right|_{t=1}, \\
\int_{0}^{1} u(s) d s= & \left.\frac{\mu}{1-\mu} I_{0+}^{\alpha+1} y(t)\right|_{t=1}-\left.\frac{\mu}{1-\mu} I_{0+}^{\alpha} y(t)\right|_{t=1} \\
& +\left.\frac{\mu}{4(1-\mu)} I_{0+}^{\alpha-1} y(t)\right|_{t=1}+\left.I_{0+}^{\alpha+1} y(t)\right|_{t=1} \\
& -\left.I_{0+}^{\alpha} y(t)\right|_{t=1}+\left.\frac{1}{2} I_{0+}^{\alpha-1} y(t)\right|_{t=1} \\
& -\left.\frac{1}{4} I_{0+}^{\alpha-1} y(t)\right|_{t=1}=\left.\frac{1}{1-\mu} I_{0+}^{\alpha+1} y(t)\right|_{t=1} \\
& -\left.\frac{1}{1-\mu} I_{0+}^{\alpha} y(t)\right|_{t=1}+\left.\frac{1}{4(1-\mu)} I_{0+}^{\alpha-1} y(t)\right|_{t=1} .
\end{aligned}
$$


This means that $u(1)=\mu \int_{0}^{1} u(s) d s$. Also, since

$$
\begin{aligned}
& u^{\prime}(0)=\left.I_{0+}^{\alpha-1} y(t)\right|_{t=0}-\left.\frac{1}{2} I_{0+}^{\alpha-1} y(t)\right|_{t=1}=-\left.\frac{1}{2} I_{0+}^{\alpha-1} y(t)\right|_{t=1}, \\
& u^{\prime}(1)=\left.I_{0+}^{\alpha-1} y(t)\right|_{t=1}-\left.\frac{1}{2} I_{0+}^{\alpha-1} y(t)\right|_{t=1}=\left.\frac{1}{2} I_{0+}^{\alpha-1} y(t)\right|_{t=1},
\end{aligned}
$$

we can see that $u^{\prime}(0)+u^{\prime}(1)=0$. Therefore, it is proved that $u$ satisfies the boundary conditions (5). The proof is completed.

Remark 3. By Lemma 2, the existence of solutions for problem (4)-(5) refers to the solvability of the integral equation (7) in $C[0,1]$.

Remark 4. As we can see from the expression

$$
\begin{aligned}
& \int_{0}^{1} G(t, s) y(s) d s=I_{0+}^{\alpha} y(t)+\left.\frac{\mu}{1-\mu} I_{0+}^{\alpha+1} y(t)\right|_{t=1} \\
& \quad-\left.\frac{1}{1-\mu} I_{0+}^{\alpha} y(t)\right|_{t=1}+\left.\left(\frac{\mu}{4(1-\mu)}+\frac{1-t}{2}\right) I_{0+}^{\alpha-1} y(t)\right|_{t=1},
\end{aligned}
$$

the function $\int_{0}^{1} G(t, s) y(s) d s$ is continuous in $[0,1]$ for any $y \in$ $C[0,1]$. Also, for any $y \in C[0,1]$, the following inequality holds:

$$
\begin{aligned}
& \left\|\int_{0}^{1} G(\cdot, s) y(s) d s\right\|=\max _{t \in[0,1]}\left|I_{0+}^{\alpha} y(t)+\frac{\mu}{1-\mu} I_{0+}^{\alpha+1} y(t)\right|_{t=1} \\
& \quad-\left.\frac{1}{1-\mu} I_{0+}^{\alpha} y(t)\right|_{t=1}+\left.\left(\frac{\mu}{4(1-\mu)}+\frac{1-t}{2}\right) I_{0+}^{\alpha-1} y(t)\right|_{t=1} \mid \\
& \quad \leq\left(\frac{1}{\Gamma(\alpha+1)}+\frac{\mu}{1-\mu} \cdot \frac{1}{\Gamma(\alpha+2)}+\frac{1}{1-\mu} \cdot \frac{1}{\Gamma(\alpha+1)}\right. \\
& \left.+\left(\frac{\mu}{4(1-\mu)}+\frac{1}{2}\right) \cdot \frac{1}{\Gamma(\alpha)}\right)\|y\|=w_{\alpha, \mu}\|y\|,
\end{aligned}
$$

where $w_{\alpha, \mu}$ is denoted as

$$
w_{\alpha, \mu}:=\frac{1}{(1-\mu) \Gamma(\alpha+1)}\left(\frac{(2-\mu)(\alpha+4)}{4}+\frac{\mu}{\alpha+1}\right) .
$$

\section{Main Results}

Define the operators $P$ and $Q$ on $C[0,1]$ as follows:

$$
\begin{aligned}
(P y)(t):= & f\left(t, \int_{0}^{1} G(t, s) y(s) d s\right), \\
(\mathrm{Q} y)(t):= & -\sum_{i=1}^{n} \lambda_{i}(t) I_{0+}^{\alpha-\alpha_{i}} y(t)-\sum_{i=1}^{m} \mu_{i}(t) I_{0+}^{\alpha-\beta_{i}} y(t) \\
& +\left.\frac{1}{2} \sum_{i=1}^{m} \frac{\mu_{i}(t) \cdot t^{1-\beta_{i}}}{\Gamma\left(2-\beta_{i}\right)} I_{0+}^{\alpha-1} y(t)\right|_{t=1}-\sigma(t) \int_{0}^{1} G(t, s) y(s) d s .
\end{aligned}
$$

Then, the integral equation (7) can be regarded as the operator equation

$$
y(t)=(P y)(t)+(Q y)(t)
$$

Lemma 5. The following hold:

(i) For any $\alpha \in \mathbf{R}^{+}$, the fractional integral operator $I_{0+}^{\alpha}$ $: C[0,1] \longrightarrow C[0,1]$ is compact.

(ii) For any $d \in C[0,1]$, the operator $A: C[0,1] \longrightarrow C[0$, 1] which is defined by

$$
(A x)(t):=d(t) \cdot x(t)
$$

is a bounded linear operator.

Proof.

(i) It is sufficient to prove that for any bounded set $\Omega:=\{u \in C[0,1]\|u\| \leq r\}, I_{0+}^{\alpha} \Omega$ is relatively compact. Obviously, we can see

$$
\begin{gathered}
\forall u \in \Omega, \\
\left|I_{0+}^{\alpha} u(t)\right| \leq \frac{1}{\Gamma(\alpha+1)}\|u\| .
\end{gathered}
$$

So, we can know that $I_{0+}^{\alpha} \Omega$ is uniformly bounded. Also, we have that for any $t_{1}, t_{2} \in[0,1]\left(t_{1}<t_{2}\right)$,

$$
\begin{aligned}
\mid I_{0+}^{\alpha} u\left(t_{1}\right)- & I_{0+}^{\alpha} u\left(t_{2}\right)|=| \frac{1}{\Gamma(\alpha)} \int_{0}^{t_{1}}\left(t_{1}-s\right)^{\alpha-1} u(s) d s \\
& -\frac{1}{\Gamma(\alpha)} \int_{0}^{t_{2}}\left(t_{2}-s\right)^{\alpha-1} u(s) d s \mid \\
& \leq \frac{1}{\Gamma(\alpha)}\left(\left|\int_{0}^{t_{1}}\left(t_{1}-s\right)^{\alpha-1} u(s) d s-\int_{0}^{t_{1}}\left(t_{2}-s\right)^{\alpha-1} u(s) d s\right|\right. \\
& \left.+\left|\int_{t_{1}}^{t_{2}}\left(t_{2}-s\right)^{\alpha-1} u(s) d s\right|\right) \\
& \leq \frac{1}{\Gamma(\alpha)}\left(\int_{0}^{t_{1}}\left(\left(t_{2}-s\right)^{\alpha-1}-\left(t_{1}-s\right)^{\alpha-1}\right) \cdot|u(s)| d s\right. \\
& \left.+\int_{t_{1}}^{t_{2}}\left(t_{2}-s\right)^{\alpha-1} \cdot|u(s)| d s\right) \leq \frac{\|u\|}{\Gamma(\alpha+1)}\left|2\left(t_{2}-t_{1}\right)^{\alpha}+t_{1}{ }^{\alpha}-t_{2}{ }^{\alpha}\right| .
\end{aligned}
$$

This yields that $I_{0+}^{\alpha} \Omega$ is equicontinuous. Therefore, using the Ascoli-Arzelà theorem, it can be easily seen that $I_{0+}^{\alpha} \Omega$ is relatively compact.

(ii) It is obvious that for any $d \in C[0,1]$,

$$
\begin{gathered}
\forall x \in C[0,1], \\
|(A x)(t)| \leq \max _{t \in[0,1]}|d(t)| \cdot\|x\| .
\end{gathered}
$$

This completes the proof of (ii). 
In this article, the following hypothesis will be used:

H1. There exist $L, l \in[0,+\infty)$ and $\sigma_{1}, \sigma_{2} \in(0,1]$ such that for any $t_{1}, t_{2} \in[0,1]$ and for any $x_{1}, x_{2} \in \mathbf{R}$,

$$
\left|f\left(t_{1}, x_{1}\right)-f\left(t_{2}, x_{2}\right)\right| \leq l\left|t_{1}-t_{2}\right|^{\sigma_{1}}+L\left|x_{1}-x_{2}\right|^{\sigma_{2}}
$$

Lemma 6. Assume that hypothesis $H 1$ holds. Then, the operator $P: C[0,1] \longrightarrow C[0,1]$ is compact.

Proof. As in the proof of Lemma 5, put $\Omega:=\{u \in C[0,1]\|\| u$ $\| \leq r\}$. Then, we have that for any $u \in \Omega$,

$$
\begin{aligned}
|(P u)(t)|= & \left|f\left(t, \int_{0}^{1} G(t, s) u(s) d s\right)\right| \leq \mid f\left(t, \int_{0}^{1} G(t, s) u(s) d s\right) \\
& -\left.f(t, 0)|+| f(t, 0)|\leq L| \int_{0}^{1} G(t, s) u(s) d s\right|^{\sigma_{2}} \\
& +|f(t, 0)| \leq L r^{\sigma_{2}} \cdot\left\|\int_{0}^{1} G(\cdot, s) d s||^{\sigma_{2}}+\right\| f(\cdot, 0) \| .
\end{aligned}
$$

This implies that $P \Omega$ is uniformly bounded.

On the other hand, we can get that for any $t_{1}, t_{2} \in[0,1]$ $\left(t_{1}<t_{2}\right)$,

$$
\begin{gathered}
\left|(P u)\left(t_{1}\right)-(P u)\left(t_{2}\right)\right|=\mid f\left(t_{1}, \int_{0}^{1} G\left(t_{1}, s\right) u(s) d s\right) \\
-f\left(t_{2}, \int_{0}^{1} G\left(t_{2}, s\right) u(s) d s\right)|\leq l| t_{1}-\left.t_{2}\right|^{\sigma_{1}} \\
+L\left|\int_{0}^{1} G\left(t_{1}, s\right) u(s) d s-\int_{0}^{1} G\left(t_{2}, s\right) u(s) d s\right|^{\sigma_{2}} .
\end{gathered}
$$

Since

$$
\begin{aligned}
& \left|\int_{0}^{1} G\left(t_{1}, s\right) u(s) d s-\int_{0}^{1} G\left(t_{2}, s\right) u(s) d s\right|=\left|I_{0+}^{\alpha} u(t)\right|_{t=t_{1}} \\
& \quad-\left.\frac{t_{1}}{2} I_{0+}^{\alpha-1} u(t)\right|_{t=1}-\left.I_{0+}^{\alpha} u(t)\right|_{t=t_{2}}+\left.\frac{t_{2}}{2} I_{0+}^{\alpha-1} u(t)\right|_{t=1} \mid \\
& \quad \leq\left|I_{0+}^{\alpha} u(t)\right|_{t=t_{1}}-\left.I_{0+}^{\alpha} u(t)\right|_{t=t_{2}} \mid \\
& \quad+\left|I_{0+}^{\alpha-1} u(t)\right|_{t=1}\left|\cdot \frac{\left|t_{1}-t_{2}\right|}{2} \leq \frac{\|u\|}{\Gamma(\alpha+1)} \cdot\right| 2\left(t_{2}-t_{1}\right)^{\alpha} \\
& +t_{1}{ }^{\alpha}-t_{2}{ }^{\alpha}\left|+\frac{\|u\|}{2 \Gamma(\alpha)} \cdot\right| t_{1}-t_{2} \mid
\end{aligned}
$$

the following inequality holds:

$$
\begin{aligned}
& \left|(P u)\left(t_{1}\right)-(P u)\left(t_{2}\right)\right| \leq l\left|t_{1}-t_{2}\right|^{\sigma_{1}} \\
& \quad+\frac{L r^{\sigma_{2}}}{\Gamma(\alpha)^{\sigma_{2}}} \cdot\left(\frac{\left|2\left(t_{2}-t_{1}\right)^{\alpha}+t_{1}{ }^{\alpha}-t_{2}{ }^{\alpha}\right|}{\alpha}+\frac{\left|t_{1}-t_{2}\right|}{2}\right)^{\sigma_{2}} .
\end{aligned}
$$

This yields that $P \Omega$ is equicontinuous. The conclusion then follows from the Ascoli-Arzelà theorem.

Lemma 7. The operator $Q: C[0,1] \longrightarrow C[0,1]$ is compact.

Proof. From Lemma 5 and the fact that the composition of the bounded linear operator and compact operator is also compact, every term of the operator $Q$ is compact. Since the sum of two compact operators is also compact, the proof is completed.

Lemma 8 (see [26]). Let $X$ be a Banach space. Assume that $\Omega$ is an open bounded subset of $X$ with $\theta \in \Omega$, and let $T: \bar{\Omega} \longrightarrow X$ be a compact operator such that

$$
\|T u\| \leq\|u\|, \quad \forall u \in \partial \Omega
$$

Then, $T$ has a fixed point in $\bar{\Omega}$.

Denote $\omega_{*}$ as follows:

$$
\begin{aligned}
\omega_{*}:= & \sum_{i=1}^{n} \frac{\left\|\lambda_{i}\right\|}{\Gamma\left(\alpha-\alpha_{i}+1\right)}+\sum_{i=1}^{m} \frac{\left\|\mu_{i}\right\|}{\Gamma\left(\alpha-\beta_{i}+1\right)} \\
& +\frac{1}{2} \sum_{i=1}^{m} \frac{\left\|\mu_{i}\right\|}{\Gamma\left(2-\beta_{i}\right) \Gamma(\alpha)}+\|\sigma\| \cdot w_{\alpha, \mu} .
\end{aligned}
$$

Here, we list more hypotheses to be used throughout this paper.

H2. $\omega_{*}<1$.

Theorem 9. Assume that hypotheses $\mathrm{H} 1$ and $\mathrm{H} 2$ hold. If the nonlinear function $f$ satisfies that

$$
\lim _{x \rightarrow 0} \max _{t \in[0,1]} \frac{f(t, x)}{x}=0,
$$

then problem (4)-(5) has at least one solution.

Proof. Since $\lim _{\|u\| \rightarrow 0}\left\|\int_{0}^{1} G(t, s) u(s) d s\right\|=0$ and $\lim _{x \rightarrow 0} \max _{t \in[0,1]} f(t, x) /$ $x=0$, we obtain

$$
\begin{gathered}
\forall d_{1}>0, \\
\exists r_{1}>0, \\
\forall u \in C[0,1] \quad\left(\|u\| \leq r_{1}\right), \\
\left\|f\left(\cdot, \int_{0}^{1} G(\cdot, s) u(s) d s\right)\right\| \leq d_{1} \cdot\|u\| .
\end{gathered}
$$


Put $d:=1-\omega_{*}$. Then, it holds that

$$
\begin{gathered}
\exists r>0, \\
\forall u \in C[0,1] \quad(\|u\| \leq r), \\
\left\|f\left(\cdot, \int_{0}^{1} G(\cdot, s) u(s) d s\right)\right\| \leq d \cdot\|u\| .
\end{gathered}
$$

Now, consider the ball $B_{r}:=\{u \in C[0,1] \mid\|u\| \leq r\}$, and for any $u_{0} \in \partial B_{r}$, estimate the norm $\left\|(P+Q) u_{0}\right\|$. Since $\| u_{0}$ $\|=r$, we can see that

$$
\begin{aligned}
& \left\|P u_{0}+Q u_{0}\right\| \leq\left\|f\left(\cdot, \int_{0}^{1} G(\cdot, s) u_{0}(s) d s\right)\right\|+\sum_{i=1}^{n} \frac{\left\|\lambda_{i}\right\| \cdot\left\|u_{0}\right\|}{\Gamma\left(\alpha-\alpha_{i}+1\right)} \\
& +\sum_{i=1}^{m} \frac{\left\|\mu_{i}\right\| \cdot\left\|u_{0}\right\|}{\Gamma\left(\alpha-\beta_{i}+1\right)}+\frac{1}{2} \sum_{i=1}^{m} \frac{\left\|\mu_{i}\right\| \cdot\left\|u_{0}\right\|}{\Gamma\left(2-\beta_{i}\right) \Gamma(\alpha)} \\
& +\|\sigma\| \cdot\left\|\int_{0}^{1} G(\cdot, s) u_{0}(s) d s\right\| \leq\left\|f\left(\cdot, \int_{0}^{1} G(\cdot, s) u_{0}(s) d s\right)\right\| \\
& +\sum_{i=1}^{n} \frac{\left\|\lambda_{i}\right\| \cdot\left\|u_{0}\right\|}{\Gamma\left(\alpha-\alpha_{i}+1\right)}+\sum_{i=1}^{m} \frac{\left\|\mu_{i} \mid\right\| \cdot\left\|u_{0}\right\|}{\Gamma\left(\alpha-\beta_{i}+1\right)}+\frac{1}{2} \sum_{i=1}^{m} \frac{\left\|\mu_{i} \mid\right\| \cdot\left\|u_{0}\right\|}{\Gamma\left(2-\beta_{i}\right) \Gamma(\alpha)} \\
& +\|\sigma\| \cdot w_{\alpha, \mu}\left\|u_{0}\right\| \leq\left(d+\omega_{*}\right) \cdot r=r=\left\|u_{0}\right\| .
\end{aligned}
$$

Therefore, the operator $P+Q$ has a fixed point in terms of Lemma 8 . This yields the conclusion.

Example 1. Consider the following fractional boundary value problem:

$$
\left\{\begin{array}{l}
{ }^{c} D_{0+}^{1.8} u(t)+0.3 t \cdot{ }^{c} D_{0+}^{1.2} u(t)+0.01 t^{2} \cdot{ }^{c} D_{0+}^{0.6} u(t)+0.2 \cdot u(t)=\sqrt{1+u^{2}(t)}-1, \\
u(1)=0.1 \int_{0}^{1} u(s) d s, u^{\prime}(0)+u^{\prime}(1)=0 .
\end{array}\right.
$$

Check that the conditions of Theorem 9 are satisfied. Putting $f(t, x)=\sqrt{1+x^{2}}-1$, it can be easily seen that

$$
\begin{gathered}
\left|f\left(t_{1}, x_{1}\right)-f\left(t_{2}, x_{2}\right)\right|=\left|\sqrt{1+x_{1}^{2}}-\sqrt{1+x_{2}^{2}}\right| \leq\left|x_{1}-x_{2}\right|, \\
\lim _{x \rightarrow 0} \max _{t \in[0,1]} \frac{f(t, x)}{x}=\lim _{x \rightarrow 0} \frac{\sqrt{1+x^{2}}-1}{x}=0 .
\end{gathered}
$$

By simple calculation, we also get $\omega_{*} \approx 0.72<1$. So, problem (41) has at least one solution.

Lemma 10 (Schaefer's fixed point theorem) (see [27]). Let $X$ be a Banach space and $A: X \longrightarrow X$ a compact operator. Then, either

(i) the equation $x=\lambda A x$ has a solution for $\lambda=1$ or

(ii) the set of all such solutions $x$, for $0<\lambda<1$, is unbounded.
Theorem 11. Assume that hypotheses H1 and H2 hold. And suppose that

(i) $\exists a>0, \exists \psi \in C([0,1] \times \mathbf{R}, \mathbf{R})$, and

$\lim _{x \rightarrow+\infty} \max _{t \in[0,1]} \frac{\psi(t, x)}{x}=0 \wedge \forall t \in[0,1], \quad \forall x \in \mathbf{R},|f(t, x)| \leq a+|\psi(t, x)|$.

(ii) $\forall t \in[0,1], x_{1} \leq x_{2} \Longrightarrow\left|f\left(t, x_{1}\right)\right| \leq\left|f\left(t, x_{2}\right)\right|$

Then, problem (4)-(5) has at least one solution.

Proof. From Lemmas 6 and 7 and hypothesis H1, the operator $P+Q$ is compact. Using condition (i), we can get

$$
\begin{gathered}
\forall d_{1}>0, \\
\exists r_{1}>0, \\
\forall x \geq r_{1}, \\
|f(t, x)| \leq a+d_{1} \cdot x .
\end{gathered}
$$

Put $d:=\left(1-\omega_{*}\right) / 2 w_{\alpha, \mu}$. Then, it follows that

$$
\begin{gathered}
\exists r>0, \\
\forall x \geq r, \\
\|f(\cdot, x)\| \leq a+d \cdot x .
\end{gathered}
$$

Now consider the set $S:=\{u \in C[0,1] \mid u=\lambda P u+\lambda Q u$, $0<\lambda<1\}$. From Remark 4, it is obvious that for any $u \in S$,

$$
\int_{0}^{1} G(\cdot, s) u(s) d s \in C[0,1]
$$

There are two cases $\left\|\int_{0}^{1} G(\cdot, s) u(s) d s\right\| \leq r$ and $\| \int_{0}^{1} G(\cdot, s) u$ $(s) d s \|>r$.

If $\left\|\int_{0}^{1} G(\cdot s) u(s) d s\right\| \leq r$, then because of condition (ii), we have

$$
\begin{aligned}
& \left|f\left(t, \int_{0}^{1} G(t, s) u(s) d s\right)\right| \leq\left|f\left(t,|| \int_{0}^{1} G(\cdot, s) u(s) d s||\right)\right| \\
& \quad \leq|f(t, r)| \leq a+d \cdot r .
\end{aligned}
$$

Thus, it follows that

$$
\begin{aligned}
& \|P u+Q u\| \leq a+d \cdot r+\left(\sum_{i=1}^{n} \frac{\left\|\lambda_{i}\right\|}{\Gamma\left(\alpha-\alpha_{i}+1\right)}+\sum_{i=1}^{m} \frac{\left\|\mu_{i}\right\|}{\Gamma\left(\alpha-\beta_{i}+1\right)}\right. \\
& \left.+\frac{1}{2} \sum_{i=1}^{m} \frac{\left\|\mu_{i}\right\|}{\Gamma\left(2-\beta_{i}\right) \Gamma(\alpha)}+\|\sigma\| \cdot w_{\alpha, \mu}\right) \cdot\|u\|=a+d \cdot r+\omega_{*}\|u\| .
\end{aligned}
$$


This yields that

$$
\|u\| \leq \frac{\lambda(a+d r)}{1-\lambda \omega_{*}} \leq \frac{a+d r}{1-\omega_{*}}
$$

If $\left\|\int_{0}^{1} G(\cdot, s) u(s) d s\right\|>r$, then as in the first case, using condition (ii) and Remark 4, we can see that

$$
\begin{gathered}
\left|f\left(t, \int_{0}^{1} G(t, s) u(s) d s\right)\right| \leq\left|f\left(t, \| \int_{0}^{1} G(\cdot, s) u(s) d s||\right)\right| \\
\leq a+d \cdot\left\|\int_{0}^{1} G(\cdot, s) u(s) d s\right\| \leq a+d \cdot w_{\alpha, \mu}\|u\| .
\end{gathered}
$$

And we obtain

$$
\begin{aligned}
& \|P u+Q u\| \leq a+d \cdot w_{\alpha, \mu}\|u\| \\
& +\left(\sum_{i=1}^{n} \frac{\left\|\lambda_{i}\right\|}{\Gamma\left(\alpha-\alpha_{i}+1\right)}+\sum_{i=1}^{m} \frac{\left\|\mu_{i}\right\|}{\Gamma\left(\alpha-\beta_{i}+1\right)}\right.
\end{aligned}
$$

$$
\begin{aligned}
& \left.+\frac{1}{2} \sum_{i=1}^{m} \frac{\left\|\mu_{i}\right\|}{\Gamma\left(2-\beta_{i}\right) \Gamma(\alpha)}+\|\sigma\| \cdot w_{\alpha, \mu}\right) \cdot\|u\| \\
& \quad=a+\left(d \cdot w_{\alpha, \mu}+\omega_{*}\right)\|u\| .
\end{aligned}
$$

Therefore, it holds that

$$
\begin{aligned}
\|u\| & \leq \frac{\lambda a}{1-\lambda\left(d \cdot w_{\alpha, \mu}+\omega_{*}\right)} \\
& \leq \frac{a}{1-d \cdot w_{\alpha, \mu}-\omega_{*}}=\frac{2 a}{1-\omega_{*}} .
\end{aligned}
$$

These inequalities (49) and (52) imply the boundedness of the set $S$. By using Lemma 10., the operator $P+Q$ has a fixed point. This completes the proof.

Example 2. Consider the boundary value problem

$$
\left\{\begin{array}{l}
{ }^{c} D_{0+}^{1.8} u(t)+0.3 t \cdot{ }^{c} D_{0+}^{1.2} u(t)+0.01 t^{2} \cdot{ }^{c} D_{0+}^{0.6} u(t)-0.8 u(t)=\sin t-\sqrt{1+u^{2}(t)}+1, \\
u(1)=0.1 \int_{0}^{1} u(s) d s, u^{\prime}(0)+u^{\prime}(1)=0 .
\end{array}\right.
$$

Problem (53) is equal to the following problem:

$$
\left\{\begin{array}{l}
{ }^{c} D_{0+}^{1.8} u(t)+0.3 t \cdot{ }^{c} D_{0+}^{1.2} u(t)+0.01 t^{2} \cdot{ }^{c} D_{0+}^{0.6} u(t)+0.2 u(t)=\sin t-\sqrt{1+u^{2}(t)}+1+u(t) \\
u(1)=0.1 \int_{0}^{1} u(s) d s, u^{\prime}(0)+u^{\prime}(1)=0
\end{array}\right.
$$

For problem (54), check that the conditions of Theorem 11 are satisfied. Putting $f(t, x)=\sin t-\sqrt{1+x^{2}}+1+x$, we can see that

$$
\begin{gathered}
\left|f\left(t_{1}, x_{1}\right)-f\left(t_{2}, x_{2}\right)\right| \leq\left|t_{1}-t_{2}\right|+2\left|x_{1}-x_{2}\right|, \\
a:=1, \\
\psi(t, x):=1+x-\sqrt{1+x^{2}}, \\
\lim _{x \rightarrow+\infty} \max _{t \in[0,1]} \frac{\psi(t, x)}{x}=0, \\
\frac{\partial f}{\partial x}(t, x)=1-\frac{x}{\sqrt{1+x^{2}}}>0 .
\end{gathered}
$$

Also, since $\omega_{*} \approx 0.72<1$, all the conditions of Theorem 11 are satisfied. So, problem (53) has at least one solution.
Theorem 12. Suppose that the following hold:

(i) There exists $L \geq 0$ such that for any $t \in[0,1]$ and for any $x_{1}, x_{2} \in \mathbf{R}$,

$$
\left|f\left(t, x_{1}\right)-f\left(t, x_{2}\right)\right| \leq L\left|x_{1}-x_{2}\right| .
$$

(ii) $q:=(L+\|\sigma\|) \cdot w_{\alpha, \mu}+\sum_{i=1}^{n}\left\|\lambda_{i}\right\| / \Gamma\left(\alpha-\alpha_{i}+1\right)+\sum_{i=1}^{m}($ $\left.\left(1 / \Gamma\left(\alpha-\beta_{i}+1\right)\right)+\left(1 / 2 \Gamma\left(2-\beta_{i}\right) \Gamma(\alpha)\right)\right)\left\|\mu_{i}\right\|<1$

Then, problem (4)-(5) has a unique solution.

Proof. Define the operator $T: C[0,1] \longrightarrow C[0,1]$ as

$$
T y(t):=(P y)(t)+(Q y)(t)
$$


Obviously, we can obtain that for any $y_{1}, y_{2} \in C[0,1]$ and for any $t \in[0,1]$,

$$
\begin{aligned}
& \left|\left(T y_{1}\right)(t)-\left(T y_{2}\right)(t)\right| \\
& \quad \leq\left|f\left(t, \int_{0}^{1} G(t, s) y_{1}(s) d s\right)-f\left(t, \int_{0}^{1} G(t, s) y_{2}(s) d s\right)\right| \\
& \quad+\sum_{i=1}^{n}\left|\lambda_{i}(t)\right| I_{0+}^{\alpha-\alpha_{i}}\left|y_{1}(t)-y_{2}(t)\right| \\
& \quad+\sum_{i=1}^{m}\left|\mu_{i}(t)\right| I_{0+}^{\alpha-\beta_{i}}\left|y_{1}(t)-y_{2}(t)\right| \\
& \quad+\frac{1}{2} \sum_{i=1}^{m} \frac{\left|\mu_{i}(t)\right|}{\Gamma\left(2-\beta_{i}\right)} I_{0+}^{\alpha-1}\left|y_{1}(t)-y_{2}(t) \|\right|_{t=1} \\
& \quad+|\sigma(t)| \cdot\left|\int_{0}^{1} G(t, s) \cdot\left(y_{1}(s)-y_{2}(s)\right) d s\right| \\
& \quad \leq\left((L+\|\sigma\|) \cdot w_{\alpha, \mu}+\sum_{i=1}^{n} \frac{\left\|\lambda_{i}\right\|}{\Gamma\left(\alpha-\alpha_{i}+1\right)}\right. \\
& \left.\quad+\sum_{i=1}^{m}\left(\frac{1}{\Gamma\left(\alpha-\beta_{i}+1\right)}+\frac{1}{2 \Gamma\left(2-\beta_{i}\right) \Gamma(\alpha)}\right)\left\|\mu_{i}\right\|\right) \\
& \quad+\left\|y_{1}-y_{2}\right\|=q\left\|y_{1}-y_{2}\right\| .
\end{aligned}
$$

This means that $T: C[0,1] \longrightarrow C[0,1]$ is a contraction operator. Therefore, the operator $T$ has a unique fixed point in terms of the Banach contraction mapping principle, and problem (4)-(5) has a unique solution.

Example 3. Consider the following boundary value problem:

$$
\left\{\begin{array}{l}
{ }^{c} D_{0+}^{1.8} u(t)+0.3 t \cdot{ }^{c} D_{0+}^{1.2} u(t)+0.01 t^{2} \cdot{ }^{c} D_{0+}^{0.6} u(t)+0.2 u(t)=\sin t+0.03 \sqrt{1+u^{2}(t)}-1, \\
u(1)=0.1 \int_{0}^{1} u(s) d s, u^{\prime}(0)+u^{\prime}(1)=0 .
\end{array}\right.
$$
that

Putting $f(t, x)=\sin t+0.03 \sqrt{1+x^{2}}-1$, we can see easily

$$
\left|f\left(t, x_{1}\right)-f\left(t, x_{2}\right)\right| \leq 0.03\left|x_{1}-x_{2}\right| \text {. }
$$

This shows that condition (i) of Theorem 12 is satisfied. On the other hand, we can get

$$
q \approx 0.78<1 \text {. }
$$

Thus, problem (59) has a unique solution.

\section{Conclusion}

In this paper, we consider the solvability of nonlinear multiorder fractional differential equations with integral and antiperiodic boundary conditions. At first, we derive an integral equation, transforming our fractional boundary value problems. Then, using some fixed point theorems such as the Banach contraction mapping principle and Schaefer's fixed point theorem, we prove the existence and uniqueness of solutions. Since the problem considered in [24] is a special case of our problem, the results of this work can be regarded as generalizations of those results.

\section{Data Availability}

No data sets are generated or analyzed during this study.

\section{Conflicts of Interest}

The authors declare that they have no competing interests.

\section{Authors' Contributions}

All authors carried out the proof and conceived of the study. All authors read and approved the final manuscript.

\section{References}

[1] S. G. Samko, A. A. Kilbas, and O. I. Marichev, Fractional Integrals and Derivatives: Theory and Applications, Gordon and Breach Science, Yverdon, Switzerland, 1993.

[2] I. Podlubny, Fractional Differential Equations, Academic Press, San Diego, 1999.

[3] R. Hilfer, Applications of Fractional Calculus in Physics, World Scientific, Singapore, 2000.

[4] K. Jong, "Existence and uniqueness of positive solutions of a kind of multi-point boundary value problems for nonlinear fractional differential equations with p-Laplacian operator," Mediterranean Journal of Mathematics, vol. 15, no. 3, 2018.

[5] K. Jong, H. Choi, and Y. Ri, "Existence of positive solutions of a class of multi-point boundary value problems for p-Laplacian fractional differential equations with singular source terms," Communications in Nonlinear Science and Numerical Simulation, vol. 72, pp. 272-281, 2019.

[6] A. A. Kilbas, H. M. Srivastava, and J. J. Trujillo, Theory and Applications of Fractional Differential Equations, vol. 204 of North-Holland Mathematics Studies, Elsevier Science B.V., Amsterdam, The Netherlands, 2006.

[7] R. P. Agarwal, B. Ahmad, and A. Alsaedi, "Fractional-order differential equations with antiperiodic boundary conditions: a survey," Boundary Value Problems, vol. 2017, no. 1, Article ID 173, 2017.

[8] A. R. Aftabizadeh, S. Aizicovici, and N. H. Pavel, "On a class of second-order anti-periodic boundary value problems," Journal of Mathematical Analysis and Applications, vol. 171, no. 2, pp. 301-320, 1992.

[9] K. Wang, "A new existence result for nonlinear first-order anti-periodic boundary value problems," Applied Mathematics Letters, vol. 21, no. 11, pp. 1149-1154, 2008.

[10] W. Wang and J. Shen, "Existence of solutions for anti-periodic boundary value problems," Nonlinear Analysis: Theory, Methods \& Applications, vol. 70, no. 2, pp. 598-605, 2009.

[11] R. P. Agarwal and B. Ahmad, "Existence theory for antiperiodic boundary value problems of fractional differential equations and inclusions," Computers \& Mathematics with Applications, vol. 62, no. 3, pp. 1200-1214, 2011.

[12] B. Ahmad and J. J. Nieto, "Anti-periodic fractional boundary value problems," Computers \& Mathematics with Applications, vol. 62 , no. 3, pp. 1150-1156, 2011. 
[13] B. Ahmad and J. Nieto, "Anti-periodic fractional boundary value problems with nonlinear term depending on lower order derivative," Fractional Calculus and Applied Analysis, vol. 15, no. 3, pp. 451-462, 2012.

[14] A. Alsaedi, B. Ahmad, N. Mohamad, and S. K. Ntouyas, "Uniqueness results for fully anti-periodic fractional boundary value problems with nonlinearity depending on lower-order derivatives," Advances in Difference Equations, vol. 2014, no. 1, Article ID 136, 2014.

[15] M. Benchohra, N. Hamidi, and J. Henderson, "Fractional differential equations with antiperiodic boundary conditions," Numerical Functional Analysis and Optimization, vol. 34, no. 4, pp. 404-414, 2013.

[16] G. Chai, "Existence results for antiperiodic boundary value problems of fractional differential equations," Advances in Difference Equations, vol. 2013, no. 1, Article ID 53, 2013.

[17] S. Choudhary and V. Daftardar-Gejji, "Nonlinear multi-order fractional differential equations with periodic/anti-periodic boundary conditions," Fractional Calculus and Applied Analysis, vol. 17, no. 2, pp. 333-347, 2014.

[18] H. Fazli and J. J. Nieto, "Fractional Langevin equation with anti-periodic boundary conditions," Chaos, Solitons \& Fractals, vol. 114, pp. 332-337, 2018.

[19] F. Wang and Z. Liu, "Antiperiodic fractional boundary value problems for nonlinear differential equations of fractional order," Advances in Difference Equations, vol. 2012, Article ID 116, 2012.

[20] G. Wang, B. Ahmad, and L. Zhang, "Existence results for nonlinear fractional differential equations with closed boundary conditions and impulses," Advances in Difference Equations, vol. 2012, no. 1, Article ID 169, 2012.

[21] M. H. Aqlan, A. Alsaedi, B. Ahmad, and J. J. Nieto, "Existence theory for sequential fractional differential equations with anti-periodic type boundary conditions," Open Mathematics, vol. 14, no. 1, pp. 723-735, 2016.

[22] M. M. Matar, "Existence of integral and anti-periodic boundary valued problem of fractional order $0<\alpha \leq 3$," Bulletin of the Malaysian Mathematical Sciences Society, vol. 40, no. 3, pp. 959-973, 2017.

[23] Y. Qiao and Z. Zhou, "Existence of solutions for a class of fractional differential equations with integral and antiperiodic boundary conditions," Boundary Value Problems, vol. 2017, no. 1, Article ID 11, 2017.

[24] Y. Xu, "Fractional boundary value problems with integral and antiperiodic boundary conditions," Bulletin of the Malaysian Mathematical Sciences Society, vol. 39, no. 2, pp. 571-587, 2016.

[25] Z. Zhou and Y. Qiao, "Solutions for a class of fractional Langevin equations with integral and antiperiodic boundary conditions," Boundary Value Problems, vol. 2018, no. 1, Article ID $152,2018$.

[26] J. Sun, Nonlinear Functional Analysis and its Application, Science Press, Beijing, 2008.

[27] D. R. Smart, Fixed Point Theorems, Cambridge University Press, London, 1980. 